\title{
Germanica
}

\section{Der Weg der Nelly Sachs von der furchtbaren zur fruchtbaren Entgrenzung}

Georges Ueberschlag

\section{OpenEdition}

1 Journals

Édition électronique

URL : http://journals.openedition.org/germanica/2493

DOI : 10.4000/germanica.2493

ISSN : 2107-0784

Éditeur

Université de Lille

\section{Édition imprimée}

Date de publication : 30 juin 1990

Pagination : 57-66

ISSN : 0984-2632

\section{Référence électronique}

Georges Ueberschlag, « Der Weg der Nelly Sachs von der furchtbaren zur fruchtbaren Entgrenzung », Germanica [Online], 7| 1990, Online erschienen am: 17 Juli 2014, abgerufen am 06 Oktober 2020. URL http://journals.openedition.org/germanica/2493; DOI : https://doi.org/10.4000/germanica.2493

Ce document a été généré automatiquement le 6 octobre 2020.

(c) Tous droits réservés 


\title{
Der Weg der Nelly Sachs von der furchtbaren zur fruchtbaren Entgrenzung
}

\author{
Georges Ueberschlag
}

1 Nelly Sachs - ein meteorenhaft erscheinender Name am Firmament der deutschen Literatur, als 1966 die schwedische Akademie ihr den Nobel-Preis der Literatur verlieh. Mehr als einer mag sich damals aber gefragt haben: wer ist sie denn? Dem grossen Publikum war sie kaum bekannt. Ihre Werke bleiben, in der Tat, auf das mindest Mögliche beschränkt, einige hundert Seiten in der Gesamtausgabe. Sie gehört nicht zu denen, schreibende monstres sacrés, deren Produktion die Bücherregale zum Biegen bringt.

Wer Nelly Sachs war, wussten 1966 nicht viele, wissen heute nicht mehr viele. Denn es wurde bald wieder still um sie, die dort oben in Stockholm, Bergsundstrand 23, beinahe 30 Jahre lang ihr Leben in einem kleinen einsamen Stübchen fristete, bis zu ihrem Tod im Jahre 1970, ohne sich der unruhvollen Gesellschaft der nach Ehre, Geld, Macht oder Ruhm haschenden Menschen anzuvertrauen. Bloss denkend und staunend betrachtete sie die Welt um sich herum, zugleich voller Menschenscheu und voller Weisheit. Der Ausblick auf den schönen Mälarsee genügte ihr.

3 In ihrem bescheidenen Leben trat kaum eine Änderung mehr ein. Einige Reisen nach Deutschland vor und nach dem Nobel, 1965 z.B. zur Entgegennahme des Friedenspreises des deutschen Buchhandels, der karge, zurückhaltende, aber nie versiegende Gedankenaustausch mit ihren schwedischen Dichterfreunden, vor allem Johannes Edfelt und Olof Lagerkrantz. Das war alles, was sie dem Leben abverlangte nach der grossen inneren und äusseren Zerrüttung, die von 1933 bis 1945, und noch einige Jahre darüber hinaus, ihr dichterisches Schicksal bestimmt hatte.

Nelly Sachs ist in einem Milieu geboren und aufgewachsen, das natürlich Grenzen kannte, natürliche Grenzen anerkannte. Als einzige Tochter des vermögenden jüdischen Fabrikanten William Sachs 1891 in Berlin geboren, hat sie eine leichte, behütete Jugend gekannt. Wie viele Mädchen aus gut bürgerlichen Kreisen, hatte sie 
Zeit, für Tanz Musik und Poesie zu schwärmen. Die Lebensregeln und Ideale ihres konservativen Milieus hat sie ohne Bedenken übernommen. Ihr innerstes Element war der Tanz, wie sie es später oft betont hat, eigentlich nicht das Dichten. Aber Tänzerin wurde man schon überhaupt nicht in solchen Kreisen, ebenso wenig wie sie ernsthaft daran denken konnte, das Abenteuer des Expressionismus, dessen soziale Infragestellung, oder den Hang ihrer Generation zu einem Randdasein in den Berliner Bohème-Kreisen zu teilen, wie z.B. Else Lasker-Schüler.

5 Der Leitstern ihres ersten dichterischen Werdens war Selma Lagerlöf, die grosse Schwedin, deren Legende von Gösta Berling sie mit 15 Jahren als Geburtstagsgeschenk erhalten hatte. In diesem Fahrwasser schrieb sie ihre ersten Gedichte, darunter zahlreiche Sonette, die Leise Melodien nannte, und die sprachlich und was die Motive betrifft, der Neuromantik sehr nahe standen.

6 Wie die Gedichte von Ricarda Huch, mit denen sie oft enge Verwandtschaft aufweisen, gehen sie Gedoch über gewöhnliche Frauenlyrik hinaus, wie man sie damals in der bürgerlichen Welt pries, und charakterisieren sie sich durch ein auffallend männliches Gepräge. Ihr Ton ist schwermütig und besonders stark elegisch für ein junges Mädchen.

$O$ die seligen Gärten der reifenden Trauben Die vergehenden, in Sehnsucht und AbendleidWährend die Schatten der Blätter noch zitternd belauben

Der Sonne Spiel mit der dunkel verwehenden Zeit.

7 so lauten z.B. einige Verse aus dem Gedicht Boccherini: Menuett, in dem schon die Vorahnung eines kommenden Abendleides anklingt.

8 Stefan Zweig, dem Nelly Sachs einige dieser Gedichte zusandte, fand es auffallend, so schrieb er, wie sie «eine ekstatisch aufsteigende Linie» aufwiesen ${ }^{1}$. Von den dichterischen Möglichkeiten der Moderne noch weit entfernt, lässt diese ekstatisch aufsteigende Linie aber doch schon ahnen, dass Nelly Sachs sich nicht immer mit den Grenzen des so schön umzäunten Jugendgärtchens abfinden wird.

9 Der ersehnte Durchbruch scheitert jedoch, 1933, an der lähmenden politischen Entwicklung, am Einbruch des tausendjährigen Reiches und an der damit verbundenen furchtbaren Entgrenzung. Alles kommt für die zarte Nelly Sachs ins Wanken, und die Verfolgunglauert und erfüllt jede Stunde mit Angst und Zittern.

10 Die fast zehn Jahre der inneren Spannung, die sie bis 1940 im national-sozialistischen Deutschland verbringt, bedeuten einen solchen Bruch in ihrem Leben, dass ihre dichterische Schöpfung fast ganz versiegt. Ihre Introvertiertheit wird angesichts der drohenden Vernichtung, die zunehmende Todesbereitschaft fordert, immer mehr betont. In ihrer persönlichen, angsterfüllten Sphäre lebend, besinnt sie sich stärker auf ihre jüdische Eigenart und vertieft sie sich in die jüdische Mystik, den Chassidismus und das Alte Testament.

11 Als ihr am 16. Mai 1940 in letzter Minute noch, zusammen mit ihrer Mutter, die Flucht nach Schweden gelingt, ist sie bereits 50 Jahre alt. Die Möglichkeit der nicht mehr erhofften Flucht und Rettung verdankt sie ihrer Freundin Gundrun Harlan und dem Prinzen Eugen Bernadotte. In Stockholm angekommen, ist sie sich zuerst kaum bewusst, was mit ihr und in ihr geschieht. Bloss eines spürt sie ganz deutlich, das Bedürfnis, zu schreiben und sich so von der angehäuften inneren Angst zu befreien. Ihre deutsche Muttersprache hatte sie ins Exil mitgenommen. Sie beginnt nicht in einer fremden Sprache zu schreiben, wie z.B. Erwin Leiser, der Freund von Brecht. Ihre 
Zugehörigkeit zur deutschen Kulturgemeinschaft bleibt für sie weiterhin eine Selbstverständlichkeit, wenn sie in langen Nächten in ihrem engen Stübchen Zwiesprache halten muss mit den gemarterten der Zeit und das Wort, der «stumme Schrei», ihr als einziges Rettungsmittel erscheint.

Aber noch ist ihr Werkzeug, die deutsche Sprache, nicht reif, das Schicksal, das selbsterlebte Schicksal der Verfolgten und Heimatlosen, denen am Ende nur die «Wohnungen des Todes» ${ }^{2}$ beschieden waren, zu schildern.

14 Aus Dankbarkeit ihrem Flüchtlingsland Schweden gegenüber, dessen Staatsangehörigkeit sie bald erwerben wird, beginnt sie, schwedische Lyrik ins Deutsche zu übersetzen. Sie tut dies aus eigenem Antrieb, nicht etwa, weil sie die Rolle einer Kulturvermittlerin spielen möchte, dazu hatte sie weder den Willen, noch die Kraft oder die Möglichkeit, auch keine Aussicht auf einen Verleger und ein paar zusätzliche Groschen. Die Beschäftigung mit der neuen schwedischen Sprache, die sie im Alter von 50 Jahren noch lernt, ist ihr ein Bedürfnis und eine innere Notwendigkeit. Ein Kern ist in ihr unangegriffen geblieben nach ihrer Flucht, von ihm aus beginnt sie die Krise zu überwinden und den Weg von der furchtbaren Entgrenzung des Terrors zur fruchtbaren Entgrenzung der lyrischen Neugeburt zu beschreiten.

Die Nahrung, die dieser Kern braucht, findet sie in der Lyrik zeitgenössischer schwedischer Dichter, von denen einige, wie Johannes Edfelt, Sivar Arner oder Olof Lagerkrantz, bald zu den wenigen zählen werden, mit denen sie sich befreundet, die ihr helfend beistehen und sie in ihren Kreis aufnehmen.

Ihre Sprache wird Nelly Sachs jetzt zu einem ganz anderen Ausdrucksmittel gestalten, unter dem doppelten Einfluss der inneren Erregung, die ausgeht von dem Bewusstsein, zu den Gejagten derZeit, zu den Heimatlosen zu gehören, mit denen sie leidet, und der äusseren Anregung die ihre Beschäftigung mit der schwedischen Lyrik bedeutet. Eine neue, persönliche Sprache entsteht, die alle bisher gekannten Formen sprengt und zu keinem literarischen Vorbild oder zu keiner Schule passt. In einem sehr sinnvollen Aufsatz über Nelly Sachs, «Das verlorene und wieder gerettete Alphabet» ${ }^{3}$, schreibt Gisela Dischner: «Den Durchbruch zu ihrem Stil verursachten nicht literarische Einflüsse und das Experimentieren mit der Sprache, sondern das Leben unter Bedrohung». Dieses Urteil entspricht, gewiss, weithin den Sachs-Interpreten, es muss aber doch nuanciert werden in seiner Einseitigkeit. Die Tatsache, dass Nelly Sachs sprachlich der Moderne fast exklusiv im Umgang mit der schwedischen Lyrik begegnet ist, spielt entscheidend mit bei ihrer lyrischen Neugeburt. Gerade zur Zeit ihres dichterischen Durchbruchs lebt sie, die an dem Expressionismus fast ganz vorüber gegangen war, in der neuen geistigen Umwelt der schwedischen Lyrik, die ihr den Sinn für die neue Bedeutung desWortes öffnet. Unter den deutschen Dichtern hatten bloss Novalis und Hölderlin eine gewisse Bedeutung für sie, vielleicht noch Paul Celan, mit dem sie befreundet war, und Kafka, in dessen Nähe man sie manchmal, aber falscherweise, stellt.

17 Der wesentliche poetische Einsatz von Nelly Sachs begann im Winter 1943-1944, also zu der Zeit, wo sie sich in ihre Übersetzungen vertieft hatte. Grenzen fallen, sprachliche und auch andere. Aber Entgrenzung ist hier keine Entgleisung. Als Übersetzerin 
schwedischer Lyrik kommt Nelly Sachs in Verbindung mit einer formalen Umwelt, die ihr die eigene Muttersprache, die im Exil aus dem üblichen Gebrauch heraus in ein neues Licht rückt - und darin liegt auch Entgrenzung - neu bewusst macht. Die neue Sprache der Dichterin trägt deutliche Schattierungen und Färbungen von der soeben übersetzten oder noch im Übersetzen begriffenen schwedischen Lyrik an sich ${ }^{4}$. Die Entgrenzung führt hier zur poetischen Selbst findung. Durch die Vermittlung des «Fremden» liess sich auch das «Eigene» in Form bringen. Dabei scheinen Begriffe wie Einfluss, kritische Rezeption kaum angebracht zu sein. Bei Nelly Sachs muss eher von einer Findung geistiger und sprachlicher Verwandtschaft und Ebenbürtigkeit gesprochen werden, wobei die vokabelnvolle, vom musikalischen Akzent durchzogene schwedische Sprache als wichtiger Ansporn mitspielte.

Dass die Wahl bei der selbstgestellten Aufgabe des Übersetzens auf die Lyrik fiel, ist beinahe selbstverständlich bei dem eigenen lyrischen Temperament der Dichterin.

Aber Wahl bedeutet auch Auswahl, und die Art und Weise, wie diese sich gestaltet, ist immer sehr aufschlussreich. Obwohl Nelly Sachs in dem Vorwort zur ersten Sammlung ihrer übersetzten Gedicht ${ }^{5}$ versucht, die Kriterien ihrer Wahl für den Leser zu bestimmen - «die markantesten Persönlichkeiten und Stilrichtungen der neueren Zeit möglichst vielseitig sprechen zu lassen» - scheint doch das persönliche Einfühlen am stärksten, ja fast ausschliesslich mitgespielt zu haben, die bewusst oder unbewusst wirkende Überzeugung, dass sie sich den Gedichten zuwenden musste, die sie im gegebenen Moment am besten nachfühlen und nachschöpfen konnte.

Sie muss sich auf jeden Fall mit diesem oder jenem Dichter, mit diesem oder jenem Gedicht, die vom Gefühl der ständigen Bedrohung umflort sind, identifizieren können. Ihre Wahl fällt auf solche, unpolitische ganz bestimmt, die sich allein der Sprache verschrieben haben, die in der Sprache eine kosmische Kraft sehen, ja die Welt überhaupt zuerstals Sprache empfinden, deren Worte «auffliegen wie Feuerzunder in einer brennenden Stadt», wie Nelly Sachs in dem schon erwähnten Vorwort schreibt. Die Lyrik ihrer Lieblinge ist zugleich verhalten wie eine Totenmesse und inbrünstig wie ein Auferstehungsgesang. Es ist eine solche, die weder richtet noch irgendwelche Bosheit kennt.

21 Natürlich kommt nur ein kleiner Teil der so umfangreichen, tiefen und schönen schwedischen Lyrik dieser Zeit in Betracht. Man sucht vergebens ein Gedicht von Vilhelm Ekelund z.B., der doch den lyrischen Frühling im Schweden des XX. Jahrhunderts eingeführt hatte und von der jungen Generation voller Enthusiasmus aufgenommen worden war. Aber Nelly Sachs geht es ja nicht darum, eine Anthologie mit all dem Systematischen, das einer solchen anhaftet, zu veröffentlichen. Der relativ kleine Teil der von ihr übersetzten Dichter kreist wesentlich um den Pol Surrealismus oder die Strömung, die man in Schweden unter dem Ausdruck «Modernismus» zusammenfasst, abgesehen von denjenigen, die wie Dan Andersson in der Romantik immer noch einen Ausweg aus der Angst-Neurose suchten.

In der labyrinthischen und gelähmten Welt dieser Dichtung sieht Nelly Sachs schon Momente ihrer eigenen Dichtung präfiguriert: die Existenzunsicherheit, die metaphysische Unruhe, das Gefühl der Fremde und die Sympathie mit den Geschlagenen, es ist eine Lyrik der Flucht und der Suche, der Suche nach dem «Land, welches nicht ist», wie der Titel eines Gedichtzyklus von Edith Södergran lautet. Katastrophen der Zeit deuten zu müssen und die gesetzten Grenzen zu übersteigen. 
Diese Dichter haben Nelly Sachs zu der Überzeugung verholfen, dass Grenzen nur da sind, Grenzen aller Art, um unsicher gemacht zu werden. "Nur wer die weissen Sommernächte oder die Dunkelheiten eines schwedischen Winters erlebt hat, schreibt sie in dem oben zitierten Vorwort, versteht in dieser Dichtung jenes Geheimnisvolle, das alle Grenzen unsicher macht».

Die übersetzten Verse enthalten Schlüsselwörter für die eigene Dichtung der Nelly Sachs, denn sie übersetzt zu einer Zeit, wo die Symbole ihrer eigenen Dichtung in ihr reifen. Man könnte sagen, dass sie übersetzt, was sie schon selbst gedichtet hat, oder auch umgekehrt, wer weiss um diese Grenzen, um das Warum dieser geheimen Wahlverwandtschaften.

Gewiss, ein eifriger Rezensent könnte eine schöne, sogenannte Plagiatliste aufstellen, ohne aber dabei das Mindeste von dieser Dichtung zu verstehen, denn wir wissen ja, dass Originalität auch aus Rezeption entstehen kann, selbst bei einer manchmal verblüffend wirkenden wörtlichen Übereinstimmung.

Es handelt sich aber bei Nelly Sachs nicht um eine zufällige oder sogar anempfundene Gemeinsamkeit. Das Wort Kongenialität würde viel besser passen, eine Kongenialität, die auf einer gemeinsamen Grundfiguration aufbaut und auf der sicheren Ahnung, dass der Dichter mehr denn je zum Aufbruch rüsten muss, «weil unser einziges Nest unsere Flügel sind», wie es in einem Vers von Erik Lindegren heisst.

Ganz bemerkenswert sind die kurzen biographischen Notizen, welche die Dichterin ihren Übersetzungen beigibt. Wie Peter Hamm treffend bemerkt, wirken sie wie verdeckte Selbstaussagen ${ }^{6}$.

Hier nur zwei Beispiele: Von Gunnar Ekelöf schreibt sie: «Er durchbricht dynamisch alle Sicherungen wie die Linien des Malers Klee... immer auf Ferneres, Weiteres hindeutend...», und von Johannes Edfelt, dem ersten schwedischen Dichter, der sie nach ihrer Flucht in Stockholm besuchte : «Mit einigen erbärmlichen abgenutzten Lappen aus der elenden Garderobe der Alltagssprache glückt es ihm, die Nacktheit des Schreckens notdürftig zu verhüllen...».

Damit definiert Nelly Sachs ihre eigene Dichtung. Selbst Erlebtes wird auf andere Dichter projiziert. Und umgekehrt wird die Reflexion über andere Dichter Element des eigenen Dichterischen. Die Motivverwandtschaft, die nur exemplarischen und keinen didaktischen Charakter hat, basiert auf beiden Seiten auf einfachen, klaren Grundwörtern, die zum Teil kontrapunktisch aufgebaut sind : Sand, Staub, Wind, Meer, Stern, Tanz, Schmetterling, Mensch, Tier, Licht, Tod, Flucht, Verwandlung... usw ${ }^{7}$. Gewiss, Motive sind Motive, Aber hier haben sie dieselbe symbolische Ballung, stützen sie sich auf dieselben fundamentalen Ausdrucksgegensätze, mit der Tendenz zur Entgrenzung, zum Ineinanderfliessen der Gegensätze.

Bienenhaft nährt sich die in ihrer Wiedergeburt befindliche Dichterin an den Blüten der schwedischen Lyrik, und dies umso leichter, da ihre eigene Muttersprache im Exil den Prozess der Verwandlung, der Entgrenzung mitgemacht hat - einer wirklich fruchtbaren Entgrenzung. "Ohne den Rückhalt der schwedischen Dichtung, schreibt Olof Lager $\mathrm{kr}$ antz, würde sie nie gewagt haben, den eigenen Eingebungen $\mathrm{zu}$ vertrauen $»^{8}$. Mit den Grundwörtern, von denen soeben die Rede war, entwickelt sie eine metaphorische Schreibweise, die gerade bei den Dichtern am stärksten auffällt, die sie mit besonderer Teilnahme las und übersetzte, nämlich bei Edfelt, Ekelöf und Lindegren. Wie diese drei, so arbeitet auch Nelly Sachs mit ihren Bildern. 

Gesetz der Metamorphose, der Flucht und der Verwaldlung wo die in Stücke geschlagenen Gefühle und Gedanken wieder zusammengesetzt, aufgesetzt werden können Aus dem Kochtopf der Sprache, die wir unter Tränen erlernten,

Ihre Bilder leben, sie stehen nicht da wie die Marmorblöcke eines Stefan George, sie entwickeln sich zu neuen Gefügen und spielen in den Bereich der Synästhesie hinein.

Es sind Metaphern, im eigentlichen Sinne des Wortes, nicht bloss Ornament, die strukturbildend geworden sind. Die schwedische Lyrik als Wegweiser in die Moderne hat ihr gezeigt, wie der Dichter die rationale Sprache des Alltags über sich hinaushebt und Bedeutungen deutet. Nach einem Wort von Lindegren hat sie gelernt «in Bildern zu denken». Aber nie, oder nur ganz selten, löst sich das Bild bei ihr in einem expressionistischen Maximalismus auf. Kein Eindruck des Gezwungenen oder Überspannten, aber doch ein Spiel mit der Sprache, «evozierende Metaphorik», wie Hellmut Geissner es ausdrückt, die jeden Schrei, jeden Exzess vermeidet.

Als Beispiel und Illustration sollen hier einige Zeilen aus dem Gedicht Chor der Wandernden angeführt werden, die zugleich das Bewusstsein ihrer schöpferischen Bindung an die schwedische Lyrik ausdrücken :

Wir Wandernde,

Unsere Wege ziehen wir als Gepäck hinter uns her - Mit einem Fetzen des Landes, darin wir

Rast hielten Sind wir bekleidet -

Aus dem Kochtopf der Sprache, die wir unter Tränen erlernten,

Ernähren wir uns... ${ }^{9}$

An Stelle des Schreis steht das Zeichen, das unumstössliche Wort.

Über die poetologischen Hinweise hinaus, die Nelly Sachs bei ihrem Umgang mit der schwedischen Lyrik dankend empfing - ihre Übersetzungsarbeit erstreckt sich übrigens über ihre ganze und kurze fünfzehnjährige Schaffensperiode - behält jedoch ihre Dichtung ein ganz eigenes Kolorit, durch ihre Verwurzelung im jüdischen Erbe, besonders im Buche «Sohar», ihre Vertrautheit mit der gnostischen Sprachbehandlung der Kabbala. Die Sprachschöpfung wird bei ihr durch das Bild des «Zungenbaums» definiert als ein organischer, in einer globalen Anschauung wurzelnder Prozess.

Eigenes und sich Anzueignendes verstand Nelly Sachs auf geistiger Ebene unter dem Ernähren wir uns...

\section{NOTES}

1. - Siehe W. Berendsohn, Nelly Sachs. Einführung in das Werk der Dichterin jüdischen Schicksals, Darmstadt 1970, S. 20.

2. - Titel des ersten Gedichtbandes von Nelly Sachs.

3. - G. Dischner, in Nelly Sachs zu Ehren, Frankfurt/Main 1966, S. 64.

4. - Siehe G. Ueberschlag, Das Wahlverhältnis der Nelly Sachs zur schwedischen Lyrik, Wissensch. Zeitschrift der M. Luther-Universität Halle, 1986/1.

5. - Von Welle und Granit, Berlin, 1947. 
6. - Siehe P. Hamm, in Nelly Sachs zu Ehren, Frankfurt/Main 1966, S. 77 ff.

7. - Diese Motivverwandtschaft, ebenso wie die formale, wurde in einem früheren Aufsatz schon untersucht und belegt. Siehe Anmerk. 4.

8. - O. Lagerkrantz, in Das Buch der Nelly Sachs, Herausg. B. Holmqvist, Frankfurt/Main 1977, S. 400.

9. - N. Sachs, In den Wohnungen des Todes, Chor der Wandernden, Suhrkamp 1965, S. 95.

\section{AUTEUR}

\section{GEORGES UEBERSCHLAG}

Université Charles de Gaulle - Lille III 